\title{
Efecto de diferentes niveles de evapotranspiración sobre área foliar, temperatura superficial, potencial hídrico y rendimiento en sorgo forrajero Effect of different levels of evapotranspiration on leaf area, temperature, water potential and yield of forage sorghum
}

\author{
Felipe Zavala-Borrego ${ }^{1}$ (D), Arturo Reyes-González ${ }^{2:}$ (D) Vicente De Paul Álvarez-Reyna1 $^{(1)}$, \\ Mario García-Carrillo ${ }^{1}$ (i) Víctor M. Rodríguez-Moreno $^{3}$ (i) y Pablo Preciado-Rangel $^{4}$ (i)
}

\footnotetext{
${ }^{1}$ Universidad Autónoma Agraria Antonio Narro Unidad Laguna. Carretera a Santa Fé y Periférico s/n, colonia Valle Verde. 27054 Torreón, Coahuila, México. ${ }^{2}$ Instituto Nacional de Investigaciones Forestales, Agrícolas y Pecuarias. Campo Experimental La Laguna. Blvd. José Santos Valdez no. 1200 pte., colonia Centro. 27440 Matamoros, Coahuila, México.

* Autor para correspondencia (reyes.arturo@inifap.gob.mx)

${ }^{3}$ Instituto Nacional de Investigaciones Forestales, Agrícolas y Pecuarias (INIFAP)- Campo Experimental Pabellón. Carretera Aguascalientes-Zacatecas km 32.5. 20660 Pabellón de Arteaga, Aguascalientes, México.

${ }^{4}$ Tecnológico Nacional de México / Instituto Tecnológico de Torreón. Carretera Torreón-San Pedro km 7.5, ejido Ana. 27170 Torreón, Coahuila, México.
}

\section{RESUMEN}

Se estima que para el año 2050 la población mundial alcance los 9.7 billones de habitantes a una tasa de crecimiento lento. Esto representa un panorama grave en cuanto al abasto del agua y a la producción de alimentos. El objetivo del presente trabajo fue determinar el efecto de diferentes niveles de evapotranspiración sobre el índice de área foliar (IAF), temperatura superficial (Ts), potencial hídrico $\left(\Psi_{\mathrm{h}}\right)$ y rendimiento en sorgo forrajero (Sorghum vulgare Pers.) bajo riego por goteo. El estudio se llevó a cabo en el verano de 2019 en las instalaciones del Campo Experimental La Laguna en Matamoros, Coahuila, México. Se evaluaron tres niveles de evapotranspiración (ET) (60, 80 y 100\%) utilizados para indicar la lámina de riego a aplicar con los tratamientos de riego por goteo y un tratamiento testigo (riego con melgas irrigadas por gravedad). Se utilizaron dos variedades de sorgo forrajero tolerantes a sequía y de alto rendimiento, Súper Sorgo (SS) y Sorgo Silo Miel (SSM). El diseño experimental utilizado fue parcelas divididas en un diseño de bloques completos al azar con cuatro repeticiones. Se observó diferencia estadística significativa $(P \leq 0.05$,
Tukey) en rendimiento verde y seco en los tratamientos de riego y variedades. Los mayores rendimientos se observaron en los tratamientos de 80 y $100 \%$ de ET, en la variedad SSM. La variedad SS presentó sus mejores rendimientos en los tratamientos con cintilla ( 80 y $100 \%$ de ET), ya que con el riego por gravedad el rendimiento disminuyó en un $26 \%$. En general las diferentes láminas de riego afectaron el IAF, Ts, $\Psi_{\mathrm{h}}$ y rendimiento, siendo el tratamiento de $60 \%$ el más afectado reduciendo su rendimiento alrededor de un $25 \%$. El volumen de agua aplicado en los tratamientos de riego con cintilla fue de 24 a $39 \%$ menor con respecto al aplicado en el riego por inundación.

Palabras clave: materia seca, riego por goteo, Sorghum vulgare Pers.

\section{SUMMARY}

By the year 2050 the world population will have reached 9.7 billion people at a slow growth rate, which represents a serious scenario in terms of water supply and food production. Thus, the objective of this research was to determine the effect of different

Cita recomendada:

Zavala-Borrego, F., Reyes-González, A., Álvarez-Reyna, V. de P., García-Carrillo, M., Rodríguez-Moreno, V. M. y Preciado-Rangel, P. (2021). Efecto de diferentes niveles de evapotranspiración sobre área foliar, temperatura superficial, potencial hídrico y rendimiento en sorgo forrajero. Terra Latinoamericana, 39, 1-14. e954. https://doi.org/10.28940/terra.v39i0.954 
evapotranspiration levels on leaf area index (LAI), surface temperature $(\mathrm{St})$, water potential $\left(\Psi_{w}\right)$ and yield in forage sorghum (Sorghum vulgare Pers.) under drip irrigation. The study was carried out in the summer of 2019 at the facilities of Campo Experimental la Laguna in Matamoros, Coahuila, México. Three evapotranspiration levels (ET) (60, 80 and $100 \%$ ) were evaluated to indicate the laminar water flow to apply with the drip irrigation treatments and a control treatment (flood irrigation), using two varieties of sorghum tolerant to drought and high yield, Super Sorgo (SS) and Sorgo Silo Miel (SSM). The randomized block experimental design was divided in plots with four replicates. A statistically significant difference ( $P \leq 0.05$, Tukey) was observed in green and dry yield between irrigation treatments and varieties. The highest yields were observed in treatments of 80 and $100 \% \mathrm{ET}$, in the SSM variety. The SS variety had its best yields in drip irrigation treatments ( 80 and $100 \%$ ET), but with flood irrigation, yield decreased by $26 \%$. In general, the different laminar irrigation flow affected the LAI, $\mathrm{S}_{\mathrm{T}}, \Psi_{\mathrm{w}}$ and yield with the treatment of $60 \%$, which was the most affected reducing its yield by $25 \%$. The volume of water applied in drip irrigation treatments was 24 to $39 \%$ less than that applied in flood irrigation.

Index words: dry matter, drip irrigation, Sorghum vulgare Pers.

\section{INTRODUCCIÓN}

Se estima que para el año 2050 la población mundial alcance los 9.7 billones de habitantes a una tasa de crecimiento lento (ONU, 2019). Esto representa un panorama grave en cuanto al abasto del agua y a la producción de alimentos, pues al contrario de lo que sucede con la población, el recurso hídrico disminuye con el paso del tiempo. Además, si a lo anterior se le suma que la agricultura, el mayor consumidor de agua en el mundo utiliza el $80 \%$ de esa agua destinada para fines de riego, no deja mucho margen para el abastecimiento de agua para consumo humano (CONAGUA, 2016).

La tasa de evapotranspiración (ET) generalmente se utiliza para estimar los requerimientos hídricos de los cultivos y es fundamental en la programación de los riegos (Reyes-González, Rivera, Delgado, Reta y Sánchez, 2019a). Diferentes porcentajes de ET relacionados con láminas de riego se han utilizado en la programación del riego de diversos cultivos como: lechuga (Lactuca sativa) (Tarqui et al., 2017), maíz (Zea mayz. L.) (Payero, Tarkalson, Irmak, Davison y Petersen, 2008), alfalfa (Medicago sativa) (GodoyAvila, Pérez, Torres, Hermosillo y Reyes, 2003), pepino (Cucumis sativus L.) (Abd-El-Mageeda y Samida, 2015), chile habanero (Capsicum chinense Jacq.) (Quintal et al., 2012), trigo (Triticum aestivum L.) (Alghory y Yazar, 2019), quinoa (Chenopodium quinoa willd.) (Bozkurt, Yazar, Alghory y Tekin, 2021), trébol rojo (Trifolium pratense) (López y Ortega, 2021), avena forrajera (Avena sativa) (Reyes-González et al., 2011), papa (Solanum tuberosum) (Patel y Rajput, 2007), algodón (Gossypium hirsitum L.) (Kalfountzos, Alexiou, Kotsopoulos, Zavakos y Vyrlas, 2006) y vid (Vitis vinifera L.) (Payán, Morales, Valdez, Martín e Ibarra, 2013). En la Comarca Lagunera son pocos los estudios que avaluaron diferentes niveles de ET en sorgo forrajero.

Por otro lado, la capacidad fotosintética de las plantas puede ser estimada utilizando el índice de área foliar (IAF), ya que la capacidad fotosintética ayuda a comprender la relación entre la producción de materia seca y el rendimiento. El IAF es útil para el desarrollo de modelos predictivos de cosecha y una forma precisa de estimar la capacidad de captura de luz del dosel; aunque la intercepción de luz tiende a saturarse en IAF $>3$ (Gao, Niu, Huang y Hou, 2013). El IAF también sirve para avaluar el desarrollo y crecimiento de los cultivos en los estudios de requerimientos hídricos y evaluaciones sobre la eficiencia bioenergética o asociarlo al daño producidos por plagas y enfermedades sobre el follaje (Acosta, Acosta, Amador y Padilla, 2008). La distribución del área foliar y la radiación dentro del dosel de la planta son algunos de los factores que afectan los procesos fotosintéticos de la planta que inciden directamente en el rendimiento del cultivo del maíz (Zea mayz. L.) forrajero, a mayor energía lumínica captada mayor será la eficiencia fotosintética de la planta (Wall y Kanemasu, 1990).

La temperatura foliar del dosel afecta los procesos fisiológicos como: difusión de gases, líquidos en la planta, solubilidad de iones, viscosidad, velocidad de transporte y la transpiración, no obstante, las fitohormonas de las plantas sirven como iniciadores y reguladores de los procesos de metabolismo, crecimiento y desarrollo, cuando la temperatura de la planta se eleva pueden morir (Fisher, Torres y Torres, 1997). Panda, Behera y Kashyap (2003) reportaron que 
la temperatura superficial de la hoja está estrechamente relacionada con la radiación solar que las hojas interceptan, a medida que se incrementa la intercepción de radiación, la hoja incrementará de manera directa su temperatura, de este modo, la temperatura de la hoja condiciona al equilibrio energético en las capas superiores de las hojas. La transpiración, la conductancia estomática y la temperatura foliar, son algunos de las diferentes variables que sirven como parámetros para estimar el estado hídrico de las plantas (Gálvez, Callejas y Reginato, 2011).

Parra, Rodríguez y González (1999) encontraron que el estrés hídrico foliar reduce el crecimiento de las hojas y restringe el crecimiento celular de la misma, al reducirse el tamaño de las hojas se reduce la capacidad fotosintética de la planta, afectando directamente los procesos fisiológicos de la planta, reduciendo la tasa de transpiración y aumentando el cierre estomático de la planta, por otra parte, la hora del día en la cual se realizan las mediciones de potencial hídrico $\left(\Psi_{\mathrm{h}}\right)$ influye en los valores de esta variable. Debido a la estrecha relación entre la humedad volumétrica del suelo y el potencial hídrico es posible utilizar $\Psi_{\mathrm{h}}$ foliar como alternativa para la programación del riego en cultivos agrícolas (García-López, Cun y Montero, 2010). El estrés hídrico de la planta incide sobre el proceso de osmorregulación de las plantas, el cual genera la capacidad para tolerar condiciones de escasez de agua y salinidad elevada, con la expresión de mecanismos adaptativos que evitan disminución de la fotosíntesis, alteraciones en la translocación, distribución de fotoasimilados y pérdidas en rendimiento, hechos significativos en el funcionamiento normal de la planta y en la productividad de los cultivos (Rodríguez, 2006).

En la Comarca Lagunera se establecieron en el 2020, 19564 ha de sorgo para forraje, 13681 ha se sembraron con agua por gravedad, 5672 ha con agua proveniente del subsuelo (rebombeo) y 211 ha se acreditaron a la siembra condicionada por las lluvias (agricultura de temporal). Se obtuvo una producción total de forraje de 852459 toneladas con un valor comercial de 633433555 pesos (SADER, 20201). Con estos indicadores, el sorgo forrajero se ha constituido como el tercer cultivo de mayor importancia en la producción de forraje en la Comarca Lagunera superado solo por los cultivos de alfalfa (Medicago sativa) (39 $343 \mathrm{ha}$ ) y maíz (Zea mayz. L.) forrajero (53 283 ha).
El objetivo de esta investigación fue evaluar el efecto de diferentes niveles de evapotranspiración sobre el índice de área foliar, temperatura foliar, potencial hídrico y rendimiento en sorgo forrajero bajo riego por goteo en un clima árido como en la Comarca Lagunera.

\section{MATERIALES Y MÉTODOS}

El estudio se llevó a cabo en las instalaciones del Campo Experimental La Laguna (CELALA) del Instituto Nacional de Investigaciones Forestales Agrícolas y Pecuarias (INIFAP) con coordenadas geográficas $102^{\circ} 00^{\prime}$ y $104^{\circ} 47^{\prime} \mathrm{O}$ y $24^{\circ} 22^{\prime}$ y $26^{\circ}$ $23^{\prime}$ N. En el CELALA se presentan veranos calurosos e inviernos fríos, se pueden alcanzar temperaturas máximas de hasta $45{ }^{\circ} \mathrm{C}$ y temperaturas mínimas de $0{ }^{\circ} \mathrm{C}$. Además, se presenta una temperatura media anual de $21.5{ }^{\circ} \mathrm{C}$, precipitación media anual de $227 \mathrm{~mm}$ y evapotranspiración (ET) media anual de 2000 milímetros.

La siembra se realizó en un suelo seco a una distancia de $13 \mathrm{~cm}$ entre semilla y $75 \mathrm{~cm}$ entre surcos para alcanzar una densidad de población de 102500 plantas por ha. Se utilizaron dos variedades de sorgo (Sorghum vulgare Pers.) resistentes a altas temperaturas, de rápido crecimiento y de ciclo vegetativo intermedio; Super Sorgo (SS) y Sorgo Silo Miel (SSM). Las dosis de fertilización que se utilizó fue 200-100-00 (NPK). Las mezclas químicas que se utilizaron fueron urea como fuente de nitrógeno $(\mathrm{N})$ y MAP para fósforo $\left(\mathrm{P}_{2} \mathrm{O}_{5}\right)$. Se aplicó todo el fosforo y la mitad de nitrógeno al momento de la siembra. La otra mitad de nitrógeno se fracciono cada dos semanas durante el ciclo del cultivo utilizando el sistema de fertirriego en el riego por goteo. Para el caso de riego por inundación con melgas irrigadas por gravedad las aplicaciones fueron; todo el fosforo y la mitad de nitrógeno al inicio de la siembra y la mitad restante de nitrógeno a los 40 días después de la siembra (DDS), la aplicación se realizó en forma manual.

Para este trabajo se estableció un diseño experimental de parcelas divididas en bloques al azar con cuatro repeticiones. Los diferentes niveles de ET (tratamientos de riego) se consideraron como parcela grande, mientras que las dos variedades de sorgo (SS y SSM) se les denominaron parcela chica. Los tratamientos de riego fueron: riego por goteo

\footnotetext{
${ }^{1}$ SADER (Secretaría de Agricultura y Desarrollo Rural). (2020). Delegación en la Región Lagunera, Sector Agropecuario, 2020. El Siglo de Torreón. Suplemento especial. Diciembre 31. 2019. (Pp. 28).
} 
subsuperficial (RGS), donde se aplicó el 60, 80 y $100 \%$ de la lámina determinada por la ET estimada por el atmómetro y un tratamiento testigo que fue el riego por inundación con melgas irrigadas por gravedad (Figura 1).

El tratamiento de $100 \%$ de ET, este se multiplicó por el coeficiente de cultivo $(\mathrm{Kc})$ correspondiente a la etapa fenológica del cultivo para calcular el consumo de agua o evapotranspiración del cultivo. Los valores de ET de referencia se tomaron de un atmómetro (ETgage, modelo A comercializado por ETgage Company Loveland, Colorado, EUA), el cual sobreestima un $2 \%$ los valores de ETo provenientes de la estación meteorológica (Reyes-González et al., 2019a). El Kc se obtuvo de la ecuación $\left(\mathrm{Kc}=1.1705^{*} \mathrm{NDVI}+0.0535\right)$ desarrollada por Reyes-González et al. (2019a) para maíz forrajero bajo riego por goteo subsuperficial. El establecimiento de las variedades de sorgo o parcelas chicas fueron cuatro surcos de $5 \mathrm{~m}$ de largo por $0.75 \mathrm{~m}$ de separación, con un área de $15 \mathrm{~m}^{2}$. La superficie total sembrada para los cuatro tratamientos fue de aproximadamente 480 metros cuadrados.

El sistema de riego por goteo subsuperficial consistió en una tubería principal de PVC y líneas regantes de tres pulgadas de diámetro. La cintilla utilizada fue Toro Aqua-Traxx enterrada a $0.25 \mathrm{~m}$, calibre 8 mil con diámetro interno de $16 \mathrm{~mm}$, espesor de pared de $0.200 \mathrm{~mm}$, emisor a $0.2 \mathrm{~m}$ y un caudal de $2.65 \mathrm{~L} \mathrm{~h}^{-1}$ por metro lineal. Las líneas regantes se establecieron a $0.75 \mathrm{~m}$ de separación. La presión de operación del sistema de riego fue de 10 libras por pulgada cuadrada (psi), intervalos entre riegos de cada tercer día. El riego para el tratamiento testigo (gravedad), se diseñó una melga de $8 \mathrm{~m}$ de ancho por $20 \mathrm{~m}$ de largo.

La medición del índice de área foliar $\mathrm{m}^{2} \mathrm{~m}^{2}$ (IAF) se realizó semanalmente, las mediciones se realizaron mediante el uso del ceptómetro PAR / LAI modelo Lp-80 de AccuPAR (Decagon Devices, Inc. Pullman, WA, EUA). La barra del ceptómetro se colocó en un ángulo de 45 grados de separación con respecto a la línea vertical del surco del cultivo. Las mediciones de la interceptación PAR se realizaron en cada parcela y en cada tratamiento en tres puntos y tres repeticiones por punto por encima y por debajo del dosel del cultivo. Las lecturas se tomaron entre las 12:00 y 14:00 horas en días despejados para minimizar la radiación difusa del cielo por las nubes (Stewart et al., 2003).

El potencial hídrico $\left(\Psi_{\mathrm{h}}\right) \mathrm{MPa}$ (Mega Pascales) se midió en días soleados. Se seleccionó la hoja que estuviera en buen estado, la cual al momento de ser cortada se cubría con tela humedecida para evitar la pérdida de agua en el traslado (Alghory y Yazar, 2019). Se realizaron dos muestreos para cada tratamiento

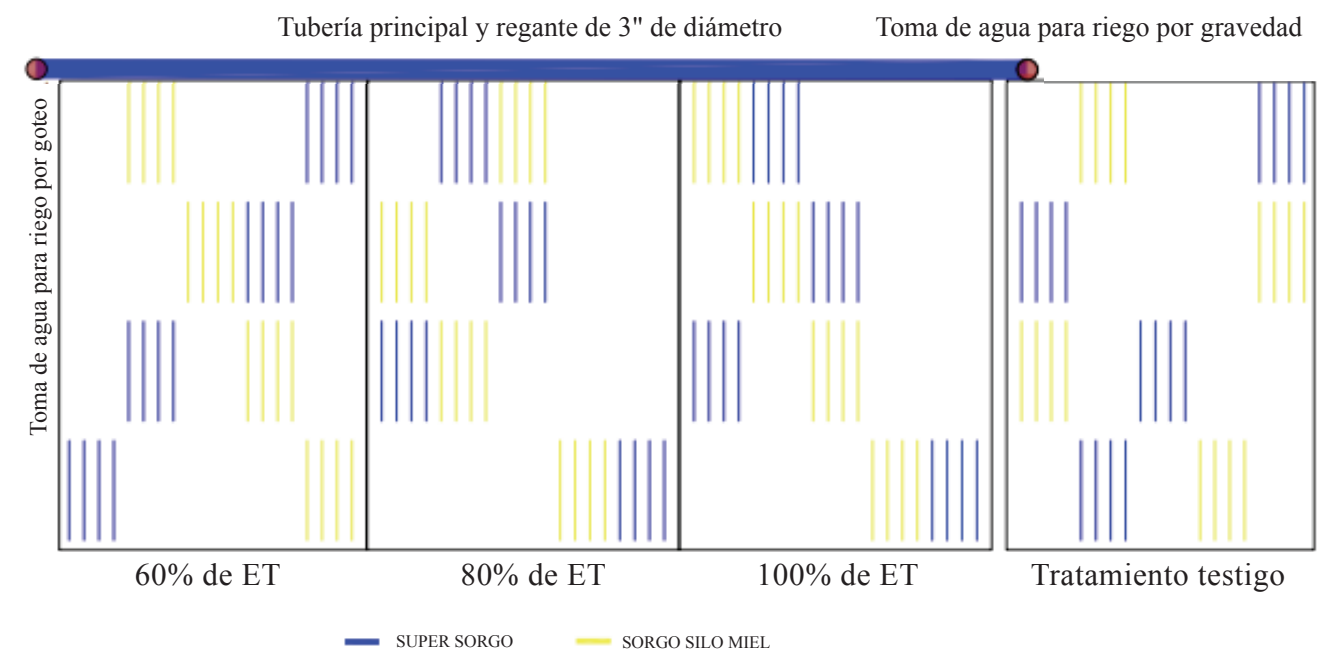

Figura 1. Croquis del experimento de dos variedades de sorgo y cuatro tratamientos de riego. Cabe señalar que en los espacios vacíos se encontraba sembrado maíz. Fuente: Elaborado con la información generada en esta investigación.

Figure 1. Sketch of the experiment of two varieties of sorghum and four irrigation treatments. It should be noted that corn was planted in empty spaces. Source: Prepared with the information generated in this research. 
dentro de cada repetición entre las 12:00 y 14:00 horas, utilizando la bomba de presión propuesta por (Scholander, Bradstreet, Hemmingsen y Hammel, 1965).

Los muestreos para la medición de la temperatura superficial ${ }^{\circ} \mathrm{C}$ (grados centígrados) de la hoja se llevaron a cabo en días completamente despejados y con velocidades de viento menor a $1 \mathrm{~m} \mathrm{~s}^{-1}$. Las temperaturas se tomaron a $25 \mathrm{~cm}$ por encima de la parte aérea del cultivo con ángulo de inclinación hacia el cultivo de 20 grados con respecto al nivel perpendicular del dosel del cultivo orientado de norte a sur. Se utilizó un termómetro digital infrarrojo modelo 42530 (Extech instruments Inc., Boston, MA, EUA), con campo de visión de 8:1 (a 8 pies $=2.4 \mathrm{~m}$ ) de distancia, el área medida es de 1 pie $(0.3 \mathrm{~m})$ de diámetro $)$. Se tomaron dos lecturas por tratamiento y repetición a partir de los 36 DDS cuando la planta de sorgo alcanzo $1.2 \mathrm{~m}$ de altura y $4.0 \mathrm{~m}^{2} \mathrm{~m}^{-2}$ de índice de área foliar.

La cosecha de las dos variedades de sorgo se realizó a los 107 DDS. La producción de forraje verde se calculó pesando tres metros lineales en los dos surcos centrales de cada parcela o unidad experimental dentro de cada tratamiento. Después de pesar la parcela útil, se tomó una muestra de $600 \mathrm{~g}$ y se secó en una estufa de aire forzado a temperatura constante de $65{ }^{\circ} \mathrm{C}$ durante 72 horas, para después estimar el rendimiento de forraje seco de las variedades en estudio y extrapolarlos a rendimiento por hectárea.

Para determinar si hubo diferencia significativa entre tratamientos de riego, rendimiento de forraje verde y seco, los datos se procesaron mediante análisis de varianza, y las medias tanto de tratamientos como de variedades se compararon utilizando la prueba de comparación de medias por el método Tukey $(P \leq 0.05)$, utilizando el programa estadístico SAS system for Windows 9.0.

\section{RESULTADOS Y DISCUSIÓN}

\section{Índice de Área Foliar (IAF)}

Al inicio de la etapa de desarrollo del sorgo, 22-57 días después de la siembra (DDS), los valores de IAF comienzan a incrementarse, después se presentan valores constantes hasta los 92 DDS y al final del ciclo los valores disminuyen ligeramente (99 DDS) (Figura 2). Los valores de IAF para el SSM fueron ligeramente superiores $(7 \%)$ a los valores registrados para el SS durante la estación de crecimiento, no se observó diferencia estadística significativa entre las dos variedades de sorgo utilizadas en este experimento.

Los valores de IAF para cada variedad de sorgo bajo diferentes niveles de ET y riego por inundación con melgas irrigadas por gravedad se presentan en la Figura 2. Los valores más bajos de IAF se presentaron en los primeros muestreos, sin embargo, para los 29 DDS el tratamiento de riego con melgas irrigadas por gravedad comenzó a arrojar los valores más bajos de todos los tratamientos de riego utilizados en el experimento y así continuo hasta los 57 DDS. Se observó diferencia significativa entre los tratamientos de riego y entre variedades de sorgo utilizadas cuando se compararon las medias de los tratamientos y variedades utilizando el método Tukey $(P \leq 0.05)$. Los valores más altos de IAF se alcanzaron a los 57 DDS y se prolongaron hasta los 78 DDS para ambas variedades, bajo riego por goteo y riego por gravedad. Durante este periodo los tratamientos de 80 y $100 \%$ de ET fueron los que presentaron los valores ligeramente altos en comparación con los otros dos tratamientos (60\% de ET y riego por gravedad). La variedad SSM presentó su valor máximo (7.92) a los 64 DDS, en el tratamiento de $80 \%$ de ET, con una deviación estándar de 0.53. Mientras que para la variedad SS el valor máximo fue de 7.24 en el tratamiento del $100 \%$ de ET, con una desviación estándar de 0.94. Después de los 86 DDS los valores de IAF para ambas variedades

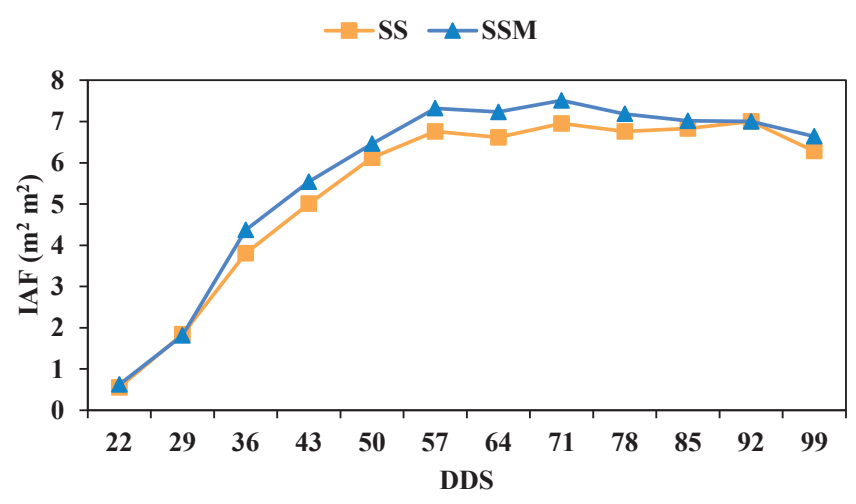

Figura 2. Comportamiento de índice de área foliar (IAF) $\left(\mathrm{m}^{2} \mathrm{~m}^{2}\right)$ de dos variedades de sorgo durante el ciclo de producción de verano 2019. Fuente: Elaborado con la información generada en esta investigación.

Figure 2. Behavior of the leaf area index (IAF) $\left(\mathrm{m}^{2} \mathrm{~m}^{2}\right)$ of two varieties of sorghum during the 2019 summer growing season. Source: Prepared with the information generated in this research. 
en los diferentes tratamientos de riego empezaron a descender ligeramente, indicando la senescencia del cultivo. En general la variedad SSM presentó valores ligeramente más altos que el SS durante el ciclo del cultivo, esto debido a que el SSM es una variedad que está adaptada a las condiciones climáticas de la región mientras que el SS se desarrolla mejor en climas tropicales (García-Berber, Ramírez y Santoscoy, 2016). Los resultados reportados en esta investigación están en el rango de valores reportados por (Carrillo y Ruiz, 2004) quienes observaron valores de IAF de 8.96 para el primer corte y 5.6 para el segundo corte en sorgo para forraje, indicando que en estos rangos hay una mayor correlación entre el IAF y la tasa de crecimiento del cultivo (Figura 3). Los resultados de IAF observados en este documento son superiores a los reportados por (Montemayor et al., 2012), quienes reportaron valores máximos de IAF (5.0) en maíz regado con riego por goteo en un experimento desarrollado en la Comarca Lagunera, México. En nuestro trabajo la restricción de agua limitó el desarrollo adecuado de las hojas, por lo que la capacidad de la planta para captar la energía luminosa se vio afectada al igual que los valores de índice de área foliar.

ॠ Super Sorgo $\bullet$ Sorgo Silo Miel
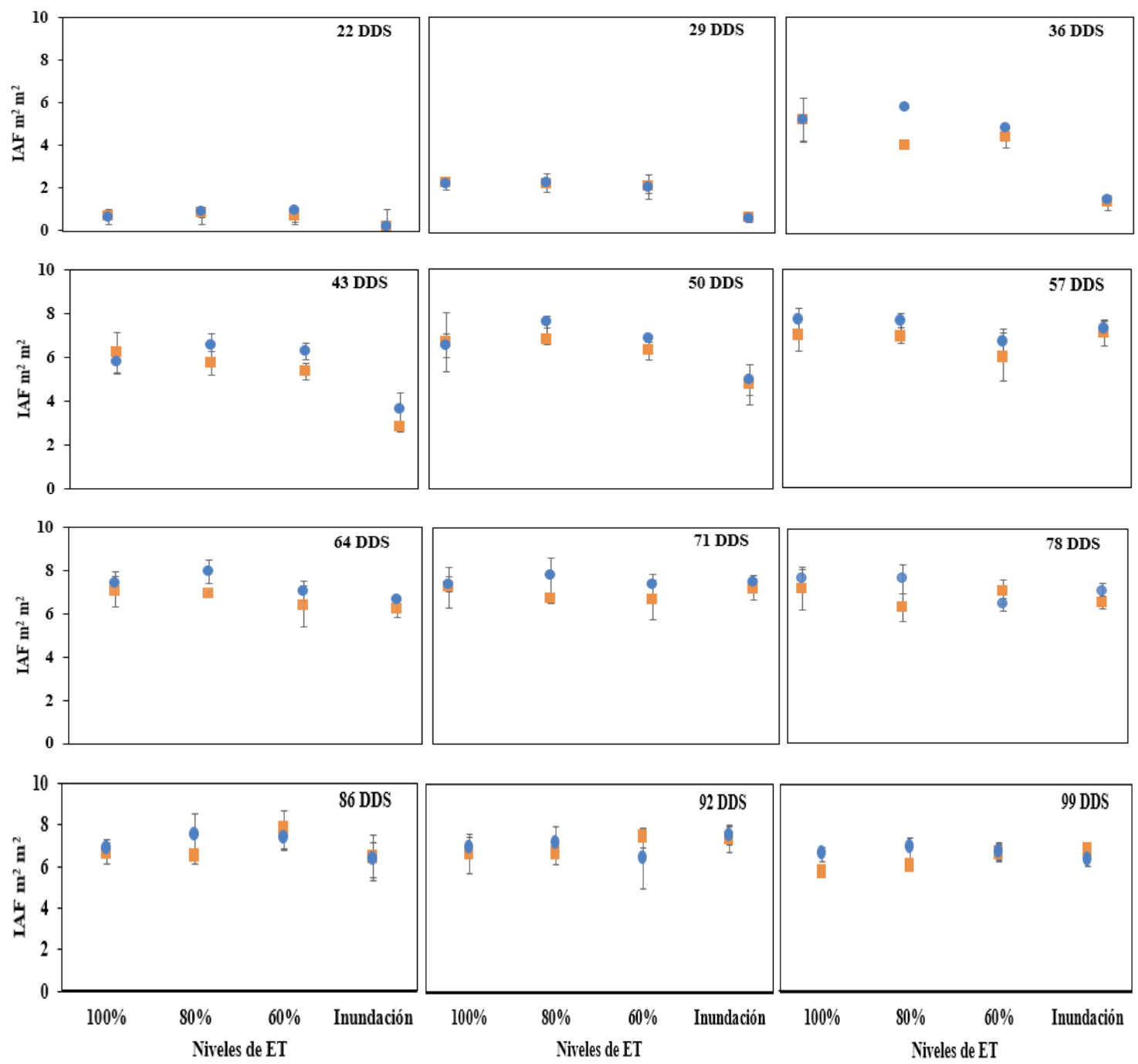

Figura 3. Valores de índice de área foliar (IAF) de dos variedades de sorgo y cuatro tratamientos de riego. Las barras verticales indican la desviación estándar. Fuente: elaborado con la información generada en esta investigación. Figure 3. Leaf area index (IAF) values of two varieties of sorghum and four irrigation treatments. Vertical bars indicate the standard deviation. Source: prepared with the information generated in this research. 


\section{Temperatura Foliar}

La temperatura foliar tuvo un comportamiento similar en las dos variedades de sorgo durante el ciclo del cultivo (Figura 4). A los 57 DDS se presentaron los valores máximos de temperatura de 35.93 y $35.18^{\circ} \mathrm{C}$ en las variedades SS y SSM, respectivamente, en el tratamiento de $60 \%$ de ET. Mientras que a los 43 DDS se presentaron los valores mínimos $28.05^{\circ} \mathrm{C}$ en la variedad SS y $28.08{ }^{\circ} \mathrm{C}$ en la variedad SSM con los tratamientos de riego de 60 y $80 \%$ de ET, respectivamente.
Los datos obtenidos en esta investigación mostraron que los valores mínimos de temperatura superficial de la hoja se presentaron en la etapa temprana de desarrollo del cultivo cuando aún no se han diferenciado los procesos fisiológicos de producción más importantes para el rendimiento de materia seca de la planta, este comportamiento y evolución de la temperatura en la planta es descrito por (Fernández y Johnston, 2006). En un estudio realizado por HimLo et al. (2020) en el cultivo de maíz, concluyeron que la temperatura superficial de la hoja estuvo influenciada por diversos factores climáticos y por

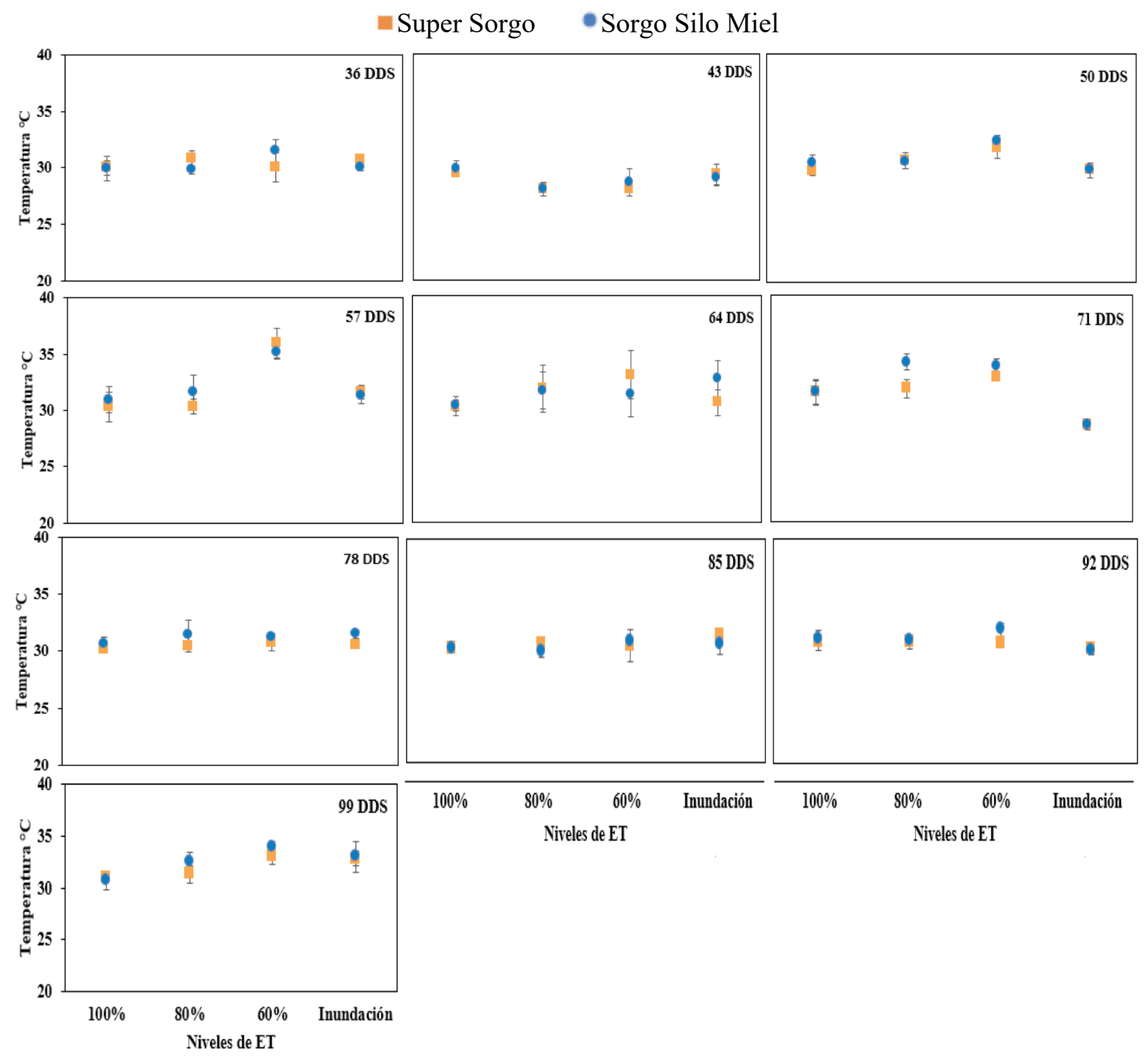

Figura 4. Temperatura foliar de dos variedades de sorgo bajo diferentes niveles de evapotranspiración (ET) e inundación. Las barras verticales indican la desviación estándar. Fuente: elaborado con la información generada en esta investigación. Figure 4. Leaf temperature of two varieties of sorghum under different levels of evapotranspiration (ET) and flooding. Vertical bars indicate the standard deviation. Source: prepared with the information generated in this research. 
diferentes regímenes de humedad, donde asociaron las temperaturas más cálidas con el déficit de humedad en el suelo. La temperatura de la superficie de la hoja y la conductancia estomática están muy relacionadas, ya que existe una relación directa que determina que al incrementarse los valores de temperatura de la hoja también se incrementa la conductancia estomática de la planta (Pino, Montalván, Vera y Ramos, 2019). De igual manera, Anderson y Kustas (2008), Durigon y de Jong van Lier (2013) y Reyes-González et al. (2019b) concluyen que, el cierre de estomas y la reducción de la transpiración, incrementan los valores de temperatura foliar, debido principalmente a los bajos niveles de humedad en la zona radical del cultivo.

\section{Potencial Hídrico $\left(\Psi_{\mathrm{h}}\right)$}

Durante el ciclo del cultivo se realizaron 12 mediciones de potencial hídrico $\left(\Psi_{\mathrm{h}}\right)$ a las dos variedades de sorgo forrajero (Figura 5). En las primeras seis mediciones el valor de $\Psi_{\mathrm{h}}$ fue de $-1.0 \mathrm{MPa}$ y para las seis siguientes el valor de $\Psi_{\mathrm{h}}$ fue de $-1.5 \mathrm{MPa}$.
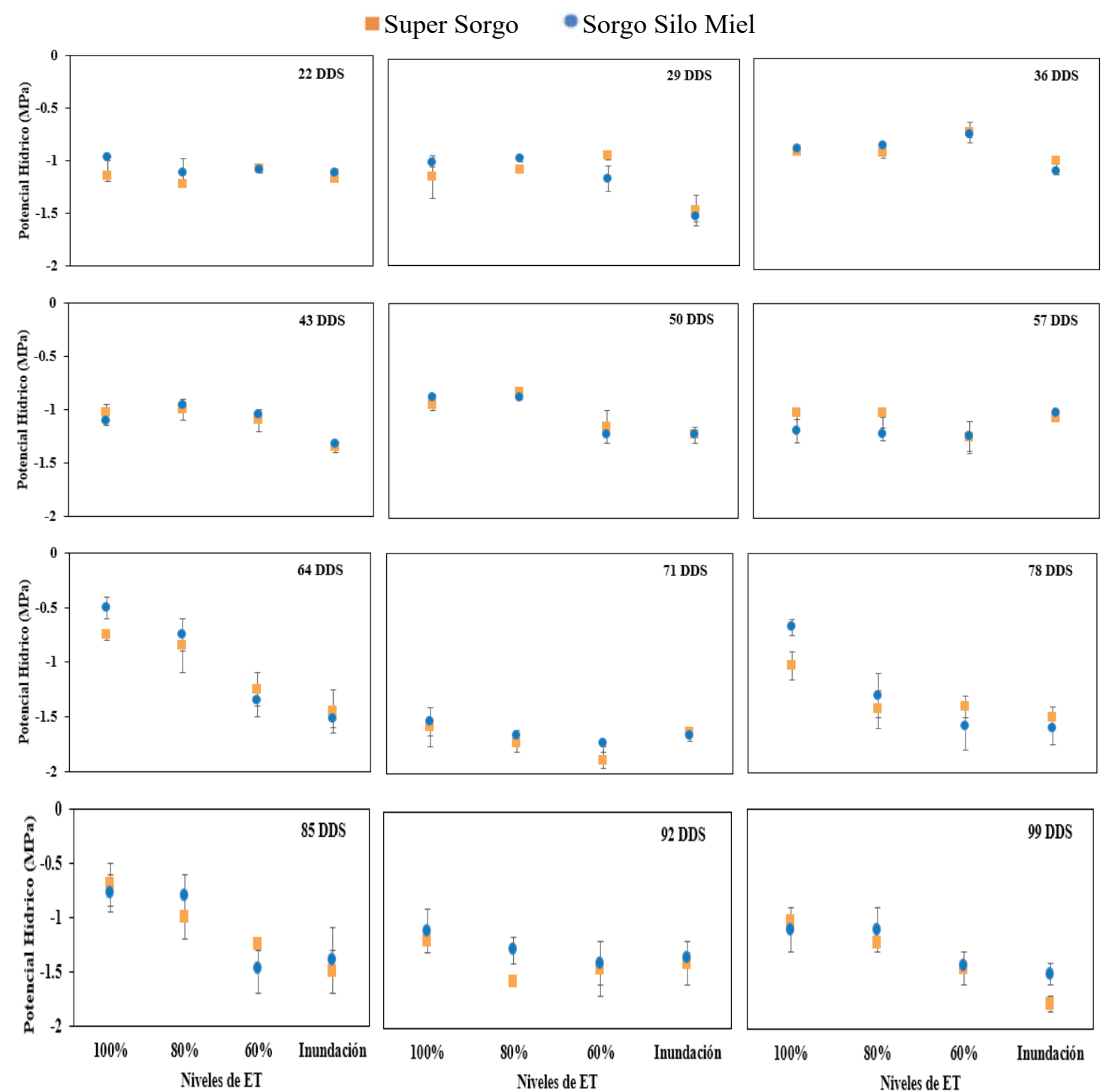

Figura 5. Potencial hídrico de dos variedades de sorgo bajo diferentes niveles de evapotranspiración (ET) e inundación. Las barras verticales indican la desviación estándar. Fuente: elaborado con la información generada en esta investigación.

Figure 5. Water potential of two varieties of sorghum under different levels of evapotranspiration (ET) and flooding. Source: prepared with the information generated in this research. 
Después de los 64 DDS los valores fueron más negativos, esto debido a que los requerimientos hídricos del cultivo fueron mayores, comparados con los valores de las primeras etapas de desarrollo. Posterior a los 64 DDS las diferencias entre los valores de potencial hídrico fue visibles entre niveles de ET o regímenes de riego, siendo el tratamiento de $60 \%$ de ET y riego con melgas irrigadas por gravedad los más negativos. Esto era de esperarse ya que después de los 57 DDS las necesidades hídricas de la planta aumentan, sin embargo, las láminas de riego fueron constantes para el tratamiento del $60 \%$ de ET. Por otro lado, a los 36 DDS se registraron los valores mínimos de $\Psi_{\mathrm{h}}$ para la variedad SS fue de $-0.7 \mathrm{MPa}$ y para la variedad SSM fue $-0.73 \mathrm{MPa}$ en el tratamiento de $60 \%$ de ET. Aunque, para este mismo tratamiento, a los 71 DDS se encontraron lo valores más negativos para la variedad SS (-1.89 MPa) y para la variedad SSM (-1.73 MPa). Estos valores contrastantes se debieron principalmente a las exigencias hídricas del cultivo de acuerdo con su etapa fenológica. En general el estado hídrico del cultivo mostró una relación directa con el tratamiento del riego aplicado: en el tratamiento donde se aplicó el $100 \%$ de ET los valores fueron menos negativos que cuando se aplicó el $60 \%$ de ET.

Los valores más negativos obtenidos en esta investigación se encuentran dentro del rango de valores para $\Psi_{\mathrm{h}}$ de -1.65 a $-2.12 \mathrm{MPa}$ descritos por
Alghory y Yazar (2019) en un experimento realizados en trigo (Trticum aestivum L.). Sin embargo, resultados semejantes fueron reportados por Reyes-González et al. (2011) los cuales oscilaron entre -0.7 y -1.9 MPa en un experimento de avena (Avena sativa) forrajera con riego por goteo en la Comarca Lagunera. Aunque valores promedios menos negativos (-0.35 $\mathrm{MPa})$ fueron reportados por Godoy-Avila et al. (2003) en el cultivo de alfalfa (Medicago sativa) irrigada con riego por goteo subsuperficial. Los valores de $\Psi_{\mathrm{h}}$ por encima de -1.3 y $-2.3 \mathrm{MPa}$ limitan la formación de nuevos brotes en la planta según Castro, López y González (2009) condiciona el crecimiento y desarrollo de la planta (Westgate y Boyer, 1985). La mejor condición hídrica de la hoja (100\% de ET) está relacionada con la disponibilidad de agua en el suelo, dando como resultado mayores rendimientos de forraje seco.

\section{Rendimiento de Forraje Verde y Seco}

El análisis estadístico para forraje verde y forraje seco resultó en diferencias significativas entre variedades $(P \leq 0.05$, Tukey) y entre tratamientos de riego ( $P \leq 0.05$, Tukey) (Cuadro 1$)$. En forraje verde hubo diferencia entre variedades $(P \leq 0.05$, Tukey), siendo la variedad SS la que alcanzó los máximos rendimientos (109 y $97 \mathrm{Mg} \mathrm{ha}^{-1}$ ) con los tratamientos de riego de 80 y $100 \%$ de $E T$, respectivamente y

Cuadro 1. Altura, forraje verde y seco para dos variedades de sorgo y diferentes niveles de evapotranspiración (ET) e inundación. Medias con letras distintas son estadísticamente diferentes $(\boldsymbol{P} \leq \mathbf{0 . 0 5})$. Fuente: elaborado con la información generada en esta investigación. Table 1. Height, green and dry forage for two varieties of sorghum and different levels of flooding evapotranspiration (ET). Means with different letters are statistically different $(\boldsymbol{P} \leq \mathbf{0 . 0 5})$. Source: prepared with the information generated in this research.

\begin{tabular}{lcccc}
\hline Niveles de ET & Variedad & Altura & Forraje verde & Forraje seco \\
\hline $100 \%$ & & $\mathrm{~m}$ & $4.0 \mathrm{a}$ & $97 \mathrm{~b}$ \\
$100 \%$ & $\mathrm{SS}$ & $3.2 \mathrm{bc}$ & $83 \mathrm{c}$ & $33 \mathrm{a}$ \\
$80 \%$ & $\mathrm{SSM}$ & $3.9 \mathrm{a}$ & $109 \mathrm{a}$ & $34 \mathrm{a}$ \\
$80 \%$ & $\mathrm{SS}$ & $3.2 \mathrm{bc}$ & $81 \mathrm{c}$ & $35 \mathrm{a}$ \\
$60 \%$ & $\mathrm{SSM}$ & $2.9 \mathrm{c}$ & $78 \mathrm{c}$ & $32 \mathrm{a}$ \\
$60 \%$ & $\mathrm{SS}$ & $2.5 \mathrm{~d}$ & $66 \mathrm{~d}$ & $24 \mathrm{c}$ \\
Inundación & $\mathrm{SSM}$ & $3.5 \mathrm{ab}$ & $76 \mathrm{c}$ & $26 \mathrm{c}$ \\
Inundación & $\mathrm{SS}$ & $2.9 \mathrm{c}$ & $77 \mathrm{c}$ & $25 \mathrm{c}$ \\
\hline
\end{tabular}

$\mathrm{SS}=$ súper sorgo. $\mathrm{SSM}=$ sorgo silo miel. $\mathrm{M}=$ metros. $\mathrm{Mg} \mathrm{ha}^{-1}=$ toneladas por hectárea.

$\mathrm{SS}=$ super sorghum. $\mathrm{SSM}=$ sorghum silo honey. $\mathrm{M}=$ meters. $\mathrm{Mg} \mathrm{ha}^{-1}=$ tons per hectare . 
los menores se registraron con la variedad SSM en el tratamiento testigo ( $77 \mathrm{Mg} \mathrm{ha}^{-1}$ ) y en el de $60 \%$ de ET $\left(66 \mathrm{Mg} \mathrm{ha}^{-1}\right)$. En cuanto a forraje seco también hubo diferencia significativa entre variedades $(P \leq 0.05$, Tukey) como fue el caso del riego por inundación, sin embargo, en los otros tratamientos no hubo diferencias $(P>0.05$, Tukey). En los tratamientos de riego hubo diferencia significativa para esta variable, siendo los tratamientos de 80 y $100 \%$ de ET los de mayor producción $\left(34 \mathrm{Mg} \mathrm{ha}^{-1}\right)$ y el de $60 \%$ de ET e inundación los de menor producción con la variedad SS (25 Mg ha $\left.{ }^{-1}\right)$. La producción final de forraje seco se vio afectada por el volumen de agua aplicado en los diferentes niveles de ET o tratamientos de riego. Para el caso de la variedad SS hubo una diferencia en rendimiento del 33\% entre los tratamientos de $100 \%$ y el de $60 \%$ de ET. Para la variedad SSM la diferencia fue del $21 \%$ entre los tratamientos donde se aplicó el $100 \%$ de ET y el tratamiento donde se aplicó el $60 \%$ de la evapotranspiración.

De acuerdo con los rendimientos de forraje seco reportado en este trabajo, se puede señalar que los rendimientos se encuentran dentro del rango de producción de materia seca de sorgo forrajero a nivel nacional. Martínez, Silva y Cuéllar (2005) mencionan que los rendimientos de forraje seco de sorgo (sorghum vulgare Pers.) forrajero oscilan entre 13 y $40 \mathrm{Mg} \mathrm{ha}^{-1}$. Sin embargo, en la presente investigación se encontraron rendimientos inferiores a los reportados por (Vargas, 2005; Bolaños, Emile y Audebert, 2012; Nava et al., 2017; Anaya, Reyes, Torres, Isidro y Martínez, 2018; Reyes-González et al., 2019b). Aunque, Ríos, Torres, Castro, Torres y Ruíz (2015) reportaron producciones superiores de 37.65 hasta $48.65 \mathrm{Mg} \mathrm{ha}^{-1}$, no obstante que estas producciones se obtuvieron en el ciclo de primavera.

\section{Evapotranspiración Acumulada (ET)}

La acumulación de ET utilizada para diferentes tratamientos de riego de sorgo forrajero se muestra en la Figura 6. En el sistema de riego por gravedad se aplicó una lámina acumulada de $710 \mathrm{~mm}$, la lámina de riego promedio aplicada por riego fue de $10 \mathrm{~cm}$ y se aplicaron siete riegos en total. En el sistema de riego por goteo se aplicaron láminas acumuladas de 432, 542 y $518 \mathrm{~mm}$ para 60,80 y $100 \%$ de ET, respectivamente.

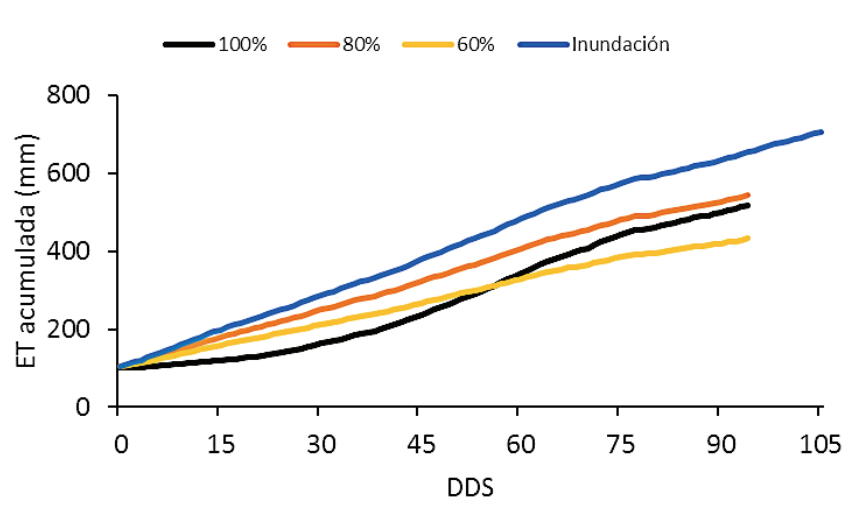

Figura 6. Evapotranspiración (ET) acumulada en diferentes niveles y riego por inundación durante el ciclo del cultivo. Fuente: elaborado con la información generada en esta investigación.

Figure 6. Evapotranspiration (ET) accumulated at different levels and flooding irrigation during the growing season. Source: prepared with the information generated in this research.

Diferentes láminas de riego fueron reportadas por diversos investigadores en cultivos forrajeros como maíz (Zea maíz. L.) (Montemayor et al., 2007), avena (Avena sativa) (Reyes-González et al., 2011) y alfalfa (Medicago sativa) (Godoy-Avila et al., 2003).

\section{Interacciones entre ET Acumulada e IAF, Temperatura, Potencial Hídrico y Rendimiento}

La relación entre ET acumulada (variable independiente) e índice de área foliar (variable dependiente) se presentan en la Figura 7a. La correlación fue de tipo polinomial de segundo orden, empezando con un efecto lineal y después cuadrático. Los coeficientes de determinación $\left(\mathrm{r}^{2}\right)$ se presentaron en un rango de 0.89 a 0.96 . Resultados similares fueron reportados por Bozkurt et al. (2021) en el cultivo de Quinoa (Chenopodium quinoa willd) quienes reportaron correlaciones de tipo polinomial entre IAF y el índice de estrés hídrico, donde los mayores valores de IAF se encontraron en el tratamiento de menor estrés por agua.

Larelaciónlineal entreET acumulada y temperatura foliar fueron bajas (Figura 7b). Como era de esperarse el tratamiento $60 \%$ de ET presentó los valores más altos de temperatura, debido a que se le suministro menor lámina de riego. Correlaciones similares fueron obtenidas por Zarazúa $\left(2013^{2}\right)$ en el cultivo de tomate (Physalis ixocarpa) quienes obtuvieron correlaciones

\footnotetext{
${ }^{2}$ Zarazúa-Bolaños, J. P. (2013). Relaciones básicas entre Evapotranspiración, Temperatura Foliar y variables climáticas. Universidad Autónoma de Querétaro. Facultad de Ingeniería. Tesis. http://hdl.handle.net/123456789/1270
} 
con coeficientes de correlación de 0.14. También Tarqui et al. (2017) presentaron baja correlación (0.49) al comparar la temperatura foliar con diferentes porcentajes de ET (75 y 100\%) en el cultivo de lechuga (Lactuca sativa) sembrada en Bolivia.

La correlación entre la ET acumulada y el potencial hídrico variaron de 0.001 hasta 0.61 (Figura 7c). Al igual que la temperatura foliar, el potencial hídrico aumento en el tratamiento con menor lámina de riego (60\% de ET). Relaciones lineales semejantes fueron reportados por Williams, Baeza y Vaughn (2012) en un viñedo de California, EUA quienes reportaron valores de potenciales hídricos más negativos en los tratamientos con menor lámina de riego. Similares comportamientos se observaron en plantas de sorgo (Sorghum bicolor L.), donde los mayores valores de potencial hídrico se presentaron con los menores valores de humedad volumétrica en el suelo (GarcíaLópez et al., 2010).

Una relación polinomial de segundo orden se presentó entre la ET acumulada y el rendimiento de forraje seco (Figura 7d). La mejor correlación se obtuvo con la variedad de sorgo SSM $\left(\mathrm{r}^{2}=0.99\right)$, mientras que con la variedad SS fue de 0.85 , lo que indica que no siempre se obtendrá los mejores rendimientos con las mayores láminas de riego. Curvas similares fueron reportadas por Bozkurt et al. (2021) con coeficientes de determinación de 0.96 para el cultivo de Quinoa (Chenopodium quinoa Willd.) regado con el sistema de riego por goteo subsuperficial. Aunque relaciones lineales fueron reportadas por Alghory y Yazar (2019) en el cultivo de trigo (Triticum eastivum L.), reportando mayores rendimientos en el cultivo menos estresado.
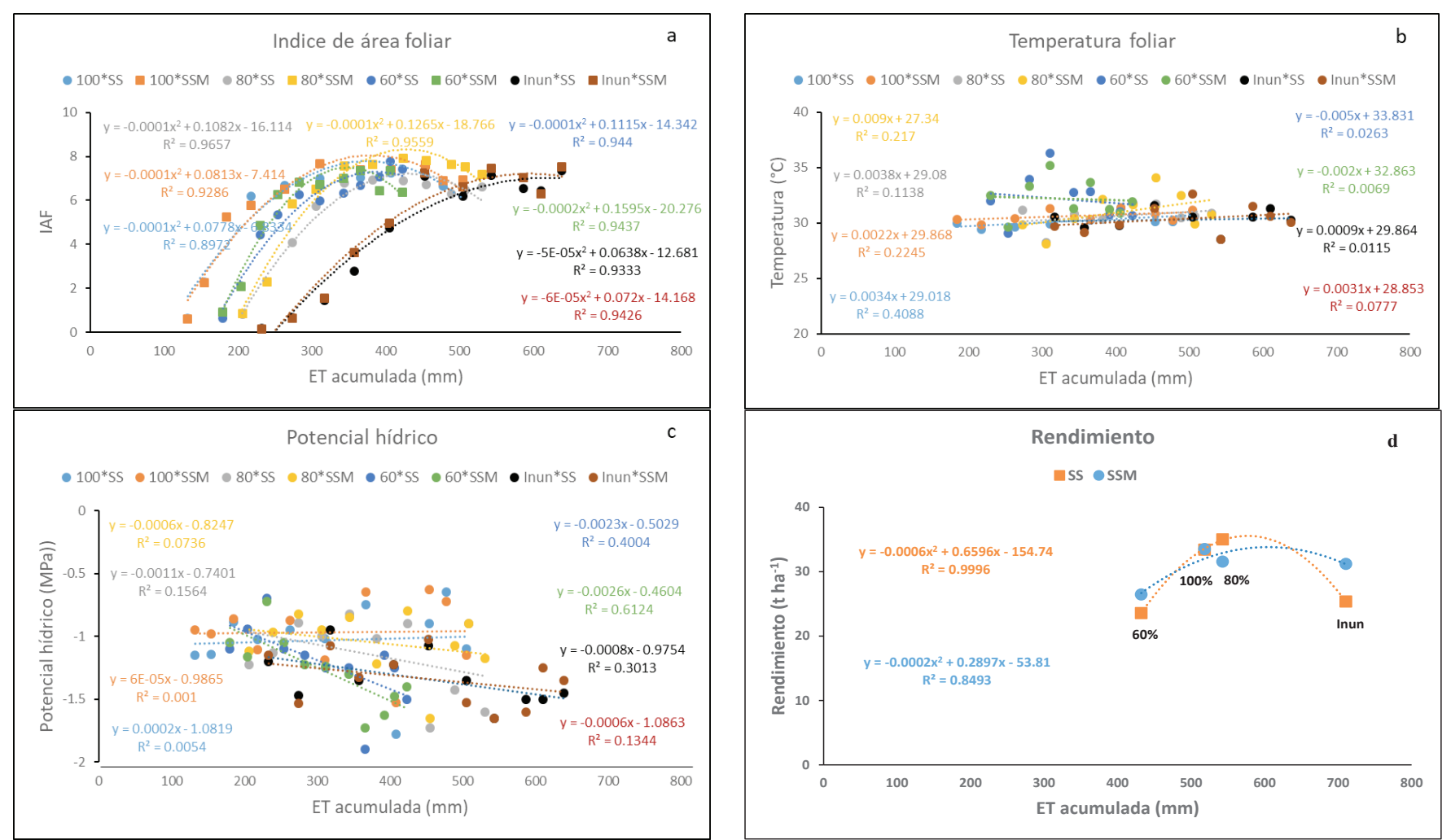

Figura 7. Interacciones entre evapotranspiración (ET) acumulada e índice de área foliar, temperatura foliar, potencial hídrico y rendimiento de dos variedades de sorgo con diferentes niveles de ET y riego por inundación. Fuente: elaborado con la información generada en esta investigación.

Figure 7. Interactions between accumulated evapotranspiration (ET) and leaf area index, leaf temperature, water potential and yield of two varieties of sorghum with different levels of ET and flood irrigation. Source: prepared with the information generated in this research. 


\section{CONCLUSIONES}

Los tratamientos de riego o diferentes niveles de evapotranspiración (ET) afectaron significativamente los valores de índice de área foliar (IAF), temperatura superficial (Ts), potencial hídrico $\left(\Psi_{\mathrm{h}}\right)$ y rendimientos en ambas variedades de sorgo durante el ciclo del cultivo. Los tratamientos de 80 y $100 \%$ de ET son los que mostraron mayor IAF, menor Ts, mínimos valores de $\Psi_{\mathrm{h}}$ y como resultado mayor rendimiento de forraje seco con respecto a los tratamientos de $60 \%$ de ET y riego por gravedad.

En lo que respecta a los rendimientos de forraje verde y seco hubo diferencia estadística significativa entre tratamientos de riego y variedades. Los tratamientos de 80 y $100 \%$ de ET mostraron los mayores rendimientos al igual que la variedad Sorgo Silo Miel (SSM) en los diferentes niveles de ET. La variedad Súper Sorgo (SS) tuvo sus mejores producciones en los tratamientos con cintilla ( 80 y $100 \%$ de ET), ya que con el riego por inundación el rendimiento disminuyo en un $26 \%$. En general las diferentes láminas de riego afectaron el IAF, Ts, $\Psi_{\mathrm{h}}$ y rendimiento, siendo el tratamiento de $60 \%$ el más afectado reduciendo el rendimiento alrededor de un $25 \%$. En cuanto al volumen de agua aplicado en los tratamientos de riego con cintilla fue de 24 a 39\% menor con respecto al aplicado en el riego por inundación con melgas irrigadas por gravedad.

De acuerdo con los resultados encontrados en este trabajo es recomendable programar los riegos tomando en cuenta la ET de referencia y los coeficientes de cultivo (Kc's) del cultivo, dependiendo de las diferentes etapas fenológicas del cultivo. Esta programación del riego es de vital importancia para eficientar el uso y manejo del agua en la agricultura.

\section{DECLARACIÓN DE ÉTICA}

No aplicable.

\section{CONSENTIMIENTO PARA PUBLICACIÓN}

No aplicable.

\section{DISPONIBILIDAD DE DATOS}

Los datos utilizados y analizados para este estudio se encuentran disponibles con el autor principal de esta redacción (Felipe Zavala Borrego) o con el autor por correspondencia (Arturo Reyes González).

\section{CONFLICTO DE INTERESES}

Los autores descritos en este documento declaran no tener ningún interés en competencia.

\section{FONDOS}

La inversión económica utilizada en esta investigación fue otorgada por el Campo Experimental La Laguna (INIFAP) de Matamoros, Coahuila, México.

\section{CONTRIBUCIÓN DE LOS AUTORES}

Desarrolló la parte esencial de este experimento, recopilación e interpretación de datos y redacción literaria: F.Z.B y A.R.G. Dirección principal y estructura de la investigación, redacción literaria y correcciones: A.R.G. Apoyó para el manejo teórico de la metodología: V.M.R.M. Ayudó en la definición de los cuestionamientos que generaron la investigación y canalización del experimento V.DP.A.R. Apoyó en la consecución de la información e interpretación de datos: P.P.R. Apoyó en la revisión ortográfica y conceptualización: M.G.C.

\section{AGRADECIMIENTOS}

Se agradece al Campo Experimental La Laguna (INIFAP) de Matamoros, Coahuila y a la Universidad Autónoma Agraria Antonio Narro - Unidad Laguna, por el trabajo conjunto que permitió el desarrollo de la investigación. Al personal técnico que ayudó en la instalación y desarrollo del experimento.

\section{LITERATURA CITADA}

Abd-El-Mageeda, T. A., \& Semida, W. M. (2015). Organo mineral fertilizer can mitigate water stress for cucumber production (Cucumis sativus L.). Agricultural Water Management, 159, 1-10. https://doi.org/10.1016/j.agwat.2015.05.020

Acosta-Díaz, E., Acosta-Gallegos, J. A., Amador-Ramírez, M. D., \& Padilla-Ramírez, J. S. (2008). Relación entre el índice de área foliar y rendimiento de del frijol boja condiciones de secano. Agricultura Técnica de México, 34(1), 13-20.

Alghory, A., \& Yazar, A. (2019). Evaluation of crop water stress index and leaf water potential for deficit irrigation management of sprinkler-irrigated wheat. Irrigation Science, 37, 61-77. https://doi.org/10.1007/s00271-018-0603-y 
Anaya-Salgado, A., Reyes-González, A., Torres-Hernández, D., Isidro Requejo, L. M., \& Martínez-Rodríguez, J. G. (2018). Producción de forraje y calidad en sorgo y mijo en condiciones agroclimática de la región Lagunera en ciclo primavera verano. En memoria de la XXIII Semana Internacional de Agronomía FAZ-UJED (pp. 237-243). Gómez Palacio, Dgo., México: FAZ-UJED.

Anderson, M., \& Kustas, W. (2008). Thermal remote sensing of drought and evapotranspiration. EOS, Transactions, American Geophysical Union, 89(26), 233-240. https://doi. org/10.1029/2008EO260001

Bolaños-Aguilar, E. D., Emile, J. C., \& Audebert, G. (2012). Rendimiento y calidad de híbridos de sorgo con y sin nervadura café. Revista Mexicana de Ciencias Agrícolas, 3(2), 441-449.

Bozkurt-Çolak, Y., Yazar, A., Alghory, A., \& Tekin, S. (2021). Evaluation of crop water stress index and leaf water potential for differentially irrigated quinoa with surface and subsurface drip systems. Irrigation Science, 39, 81-100. https://doi. org/10.1007/s00271-020-00681-4

Castro-Montes, I., López-Peralta, M. C., \& González-Hernández, V. A. (2009). Evaluación morfo-fisiológica de brotes de maíz sometidos a selección in vitro bajo estrés osmótico. Montecillo Texcoco México. Revista Fitotecnia Mexicana, 32(4), 281-288.

CONAGUA (Comisión Nacional del Agua). (2016). Clasificación de la intensidad de sequía. Consultado el 08 de diciembre, 2019, desde http://smn1.conagua.gob.mx/index.php?option=com content\&view=article \&id=237: clasiicacion-de-la-severidadde-la-sequia\&catid=16:general

Carrillo, J. C., \& Ruiz, J. (2004). Producción de forraje en sorgo y mijo; variables de crecimiento. Agronomía Mesoamericana, 15(1), 69-76.

Durigon, A., \& de Jong van Lier, Q. (2013). Canopy temperature versus soil water pressure head for the prediction of crop water stress. Agricultural Water Management, 127, 1-6. https://doi.org/10.1016/j.agwat.2013.05.014

Fernández, G., \& Johnston, M. (2006). Crecimiento y temperatura. En F. A. Squeo, \& L. Cardemil (Eds.). Fisiología vegetal (1-28). Chile: Ediciones Universidad de La Serena. ISBN: 970-625-024-7.

Fisher, G., Torres-Carbajal, F., \& Torres-Bazurto, J. (1997). Efecto de la temperatura del suelo sobre la planta. 1. Crecimiento y desarrollo. Revista Comalfi, 24(3), 78-92.

Gálvez-Pavez, R., Callejas-Rodríguez, R., \& Reginato-Meza, G. (2011). Comparación de la cámara de presión tipo Scholander modelo Pump-up respecto a la cámara de presión tradicional en vides de mesa. IDESIA, 29(2), 175-179. http://dx.doi. org/10.4067/S0718-34292011000200023

Gao, S., Niu, Z., Huang, N., \& Hou, X. (2013). Estimating the leaf area index, height and biomass of maize using $\mathrm{Hj}-1$ and RADARSAT-2. International Journal of Applied Earth Observation and Geoinformation, 24, 1-8. https://doi. org/10.1016/j.jag.2013.02.002

García-Berber, A., Ramírez-Valencia, F. J., \& Santoscoy-Padilla, A. (2016). Super Sorgo: El sorgo dulce como una alternativa rentable para algunas regiones del trópico mexicano. [Folleto Técnico No. 15, INIFAP]. Tecomán, Colima, México.
García-López, A., Cun-González, R., \& Montero-San Jose, L. (2010). Efecto de la hora del día en el potencial hídrico foliar del sorgo y su relación con la humedad en el suelo. Revista Ciencia Técnicas Agropecuarias, 19(3), 7-11.

Godoy-Avila, C., Pérez-Gutiérrez, A., Torres E., C. A., Hermosillo, L. J., \& Reyes J., I. (2003). Uso de agua, producción de forraje y relaciones hídricas en alfalfa con riego por goteo subsuperficial. Revista Agrociencia, 3(2), 107-115.

Him-Lo, T., Rudnick, D. R., DeJonge, K. C., Bai, G., NjukyNakabuye, H., Katimbo, A., ... Heeren, D. M. (2020). Differences in soil water changes and canopy temperature under varying water $\times$ nitrogen sufficiency for maize. Irrigation Science, 38, 519-534. https://doi.org/10.1007/ s00271-020-00683-2

Kalfountzos, D., Alexiou, I., Kotsopoulos, S., Zavakos, G., \& Vyrlas. P. (2006). Effect of subsurface drip irrigation on cotton plantations. Water Resource Management, 21, 13411351. https://doi.org/10.1007/s11269-006-9085-4

López-Olivari, R., \& Ortega-Klose, F. (2021). Response of red clover to deficit irrigation: dry matter yield, populations, and irrigation water use efficiency in southern Chile. Irrigation Science, 39, 173-189. https://doi.org/10.1007/s00271-02000693-0

Martínez-Villa, J., Silva-Sáenz, R. A., \& Cuéllar-Villareal, E. J. (2005). Guía para cultivar sorgo forrajero de riego para pastoreo, verdeo y henificado en el norte y centro de Coahuila. [Folleto para productores No. 7, INIFAP]. Coahuila, México.

Montemayor-Trejo, J. A., Lara-Míreles, J. L., Woo-Reza, J. L., Munguía-López, J., Rivera-González, M., \& TrucíosCasiano, R. (2012). Producción de maíz forrajero (Zea mays L.) en tres sistemas de irrigación en la Comarca Lagunera de Coahuila y Durango, México. Agrociencia, 46(3), 267-278.

Montemayor-Trejo, J. A., Olague-Ramírez, J., Fortis-Hernández, M., Sam-Bravo, R., Leos-Rodríguez, J. A., Salazar-Sosa, E., ... Chavaría-Galicia, J. A. (2007). Consumo de agua en maíz forrajero con riego subsuperficial. Terra Latinoamericana, 25(2), 163-168.

Nava-Berumen, C. A., Rosales-Serna, R., Jiménez-Ocampo, R., Carrete-Carreón, F. O., Domínguez-Martínez, P. A., \& Murillo-Ortiz, M. (2017). Rendimiento y valor nutricional de tres variedades de sorgo dulce cultivadas en cuatro ambientes de Durango. Revista Mexicana de Ciencias Pecuarias, 8(2), 147-155. https://doi.org/10.22319/rmcp.v8i2.4426

ONU (Organización de las Naciones Unidas). (2019). Department of Economic and Social Affairs. Consultado el 05 de febrero, 2020, desde http://www.un.org/development/desa/en/news/ population/word-population-prospect-2019.html

Panda, R. K., Behera, S. K., \& Kashyap, P. S. (2003). Effective management of irrigation water for wheat under stressed conditions. Agricultural Water Management, 63(1), 37-56. https://doi.org/10.1016/S0378-3774(03)00099-4

Parra-Quezada, R. A., Rodríguez-Ontiveros, J. L., \& GonzálezHernández, V. A. (1999) Transpiración, potencial hídrico y prolina en zarzamora bajo déficit hídrico. Terra, 17(2), 125-130.

Patel, N., \& Rajput, T. B. S. (2007). Effect of drip tape placement depth and irrigation level on yield of potato. Agricultural Water Management, 88(1-3), 209-223. https://doi.org/10.1016/j. agwat.2006.10.017 
Payán-Ochoa, S., Morales-Maza, A., Valdez-Gascón, B., MartínRivera, M. H., \& Ibarra-Flores, F. A. (2013). Programación del riego en vid (Vitis vinifera L.) de mesa perlette y sugraone con sensores de humedad. Revista Chapingo Serie Horticultura, 19(2), 163-172. https://doi.org/10.5154/r.rchsh.2012.04.024

Payero, J. O., Tarkalson, D. D., Irmak, S., Davison, D., \& Petersen, J. L. (2008). Effect of irrigation amounts applied with subsurface drip irrigation on corn evapotranspiration, yield, water use efficiency, and dry matter production in a semiarid climate. Agricultural Water Management, 95(8), 895-908. https://doi.org/10.1016/j.agwat.2008.02.015

Pino V., E., Montalván D., I., Vera M., A., \& Ramos F., L. (2019). La conductancia estomática y su relación con la temperatura foliar y humedad del suelo en el cultivo del olivo (Olea europaea L.), en periodo de maduración de frutos, en zonas áridas. La Yarada, Tacna, Perú. IDESIA, 37(4), 55-64. http:// dx.doi.org/10.4067/S0718-34292019000400055

Quintal-Ortiz, W. C., Pérez-Gutiérrez, A., Latournerie-Moreno, L., May-Lara, C., Ruiz-Sánchez, E., \& Martínez-Chacón, A. J. (2012). Uso de agua, potencial hídrico y rendimiento en chile habanero (Capsicum chinense Jacq.). Revista Fitotecnia Mexicana, 35(2), 155-160.

Reyes-González, A., Rivera-González, M., Delgado-Ramírez, G., Reta-Sánchez, D. G., \& Sánchez-Duarte, J. I. (2019a). Programación del riego mediante el uso de un atmómetro. AGROFAZ - Journal of Environmental and Agroecological Science, 1(1), 68-79.

Reyes-González, A., Reta-Sánchez, D. G., Sánchez-Duarte, J. I., Ochoa-Martínez, E., Rodríguez-Hernández, K., \& PreciadoRangel, P. (2019b). Estimación de la evapotranspiración de maíz forrajero apoyada con sensores remotos y mediciones in situ. Terra Latinoamericana, 37(3), 279-290. https://doi. org/10.28940/terra.v37i3.485

Reyes-González, A., Martínez-Rodríguez, J. G., PalomoRodríguez, M., Faz-Contreras, R., Cruz-Chaires, J. J., \& Sánchez-Duarte, J. I. (2011). Producción de avena forrajera con riego por goteo subsuperficial en la Comarca Lagunera. En memoria de la XXIII Semana Internacional de Agronomía FAZ-UJED (pp. 189-195). Gómez Palacio, Dgo., México: FAZ-UJED.
Ríos-Flores, J. L., Torres-Moreno, M., Castro-Franco, R., TorresMoreno, M. A., \& Ruiz-Torres, J. (2015). Determinación de la huella hídrica azul en los cultivos forrajeros del DR017, Comarca Lagunera, México. Revista de la Facultad de Ciencias Agrarias, 47(1), 93-107.

Rodríguez-Pérez, L. (2006). Implicaciones fisiológicas de la osmorregulación en plantas. Agronomía Colombiana, 24(1), 28-37.

Scholander, P. F., Bradstreet, E. D., Hemmingsen, E. A., \& Hammel, H. T. (1965). Sap pressure in vascular plants Negative hydrostatic pressure can be measured in plants. Proceedings of the National Academy of Sciences, 148(3668), 339-346. https://doi.org/10.1126/science.148.3668.339

Stewart, D. W., Costa, C., Dwyer, L. M., Smith, D. L., Hamilton, R. I., \& Ma, B. L. (2003). Canopy structure, light interception, and photosynthesis in maize. Agronomy Journal, 95(6), 14651474. https://doi.org/10.2134/agronj2003.1465

Tarqui-Delgado, M., Chipana-Rivera, R., Mena-Herrera, F. C., Quino-Luna, J. J., Tallacagua-Terrazas, R., \& GutiérrezVillalobos, S. (2017). Índice de estrés hídrico del cultivo de lechuga (Lactuca sativa), mediante termometría infrarroja a diferentes láminas de riego. Revista de Investigación e Innovación Agropecuaria y de Recursos Naturales, 4(1), 7-18.

Vargas-Rodríguez, C. F. (2005). Valoración nutricional y degradabilidad ruminal de genotipos de sorgo forrajero (Sorghum sp). Agronomía Mesoamericana, 16(2), 215-223.

Wall, G. W., \& Kanemasu, E. T. (1990). Carbon dioxide exchange rates in wheat canopies. II. Photosynthetic and phytomass production efficiencies. Agricultural and Forest Meteorology, 49(2), 103-122. https://doi.org/10.1016/0168-1923(90)90045-8

Westgate, M. E., \& Boyer, J. S. (1985). Osmotic adjustment and the inhibition of leaf, root, stem, and silk growth at low water potentials in maize. Planta, 164(4), 540-549. https://doi. org/10.1007/BF00395973

Williams, L. E., Baeza, P., \& Vaughn, P. (2012). Midday measurements of leaf water potential and stomatal conductance are highly correlated with daily water use of Thompson Seedless grapevines. Irrigation Science, 30, 201212. https://doi.org/10.1007/s00271-011-0276-2 